\title{
INVEST
}

INVEST Working Papers 36/2021

\section{From sequences to variables - Rethinking the relationship between sequences and outcomes}

Satu Helske Jouni Helske

Guilherme K. Chihaya 
The Inequalities, Interventions, and New Welfare State (INVEST) ecosystem brings together the efforts of over 200 researchers and advances cross-disciplinary research. It forms an international collaboration platform that connects researchers, practitioners, public organisations, third sector organisations, companies, students, families and other citizens that work with us in our mission to increase the wellbeing of the Finnish society during childhood, youth and early adulthood. The INVEST ecosystem can be harnessed to solve welfare state and public sector problems and provide greater insights to governments as they form policy. It is involved in large-scale policy reforms. INVEST is funded by the Academy of Finland Flagship Programme and the host institutions and affiliates multiple projects funded by various external research funders. The INVEST working paper series has subseries for the long-term projects funded by the Strategic Research Council (SRC). 
From sequences to variables - Rethinking the relationship between sequences and outcomes

Key words: Sequence analysis, cluster analysis, typology, representativeness, life course 


\begin{abstract}
Sequence analysis (SA) has gained increasing interest in social sciences for the holistic analysis of life course and other longitudinal data. The usual approach is to construct sequences, calculate dissimilarities, group similar sequences with cluster analysis, and use cluster membership as a dependent or independent variable in a regression model.

This approach may be problematic as cluster memberships are assumed to be fixed known characteristics of the subjects in subsequent analyses. Furthermore, often it is more reasonable to assume that individual sequences are mixtures of multiple ideal types rather than equal members of some group. Failing to account for uncertain and mixed memberships may lead to wrong conclusions about the nature of the studied relationships.

In this paper, we bring forward and discuss the problems of the "traditional" use of SA clusters as variables and compare four approaches for creating explanatory variables from sequence dissimilarities using different types of data. We conduct simulation and empirical studies, demonstrating the importance of considering how sequences and outcomes are related and the need to adjust analyses accordingly. In many typical social science applications, the traditional approach is prone to result in wrong conclusions and so-called similarity-based approaches such as representativeness should be preferred.
\end{abstract}




\section{Introduction}

Over the past few decades, sequence analysis (SA) has gained more and more interest in social sciences for the holistic analysis of life course and other longitudinal data. The usual approach is to construct sequences, calculate pairwise dissimilarities, and then use a clustering algorithm on the dissimilarities for finding groups of similar sequences. Typically, these clusters are then described and interpreted as typologies. Increasingly, researchers are interested in analysing the relationships between sequences and other characteristics, usually by using cluster membership as a dependent or independent variable in a linear or nonlinear regression model.

Almost unanimously, the clustering methods used in the SA context have been hard or crisp clustering algorithms such as Ward's method or partitioning around medoids (PAM). These algorithms find a partitioning where each sequence belongs to one cluster and one cluster only, which easily translates into a categorical variable with internally homogeneous and mutually exclusive groups. Applications using cluster membership as an observed characteristic of the units of analysis in regression models are also common (e.g. ??). This approach is often problematic because the implicit assumption is that cluster membership is a fixed and known characteristic of an individual (or other subject), even though there is considerable uncertainty in clustering solutions due to various possibilities of choosing (dis)similarity measures, clustering algorithms, and the number of clusters. Furthermore, individual sequences might be mixtures of two or more ideal types or distant from all ideal types, making the whole concept of classification into clear or true clusters problematic. Failing to account for uncertain and mixed memberships may lead to wrong conclusions about the existence and nature of the studied relationships.

Our aim is to bring forward and discuss the potential problems of the "traditional" approach of creating variables from SA clusters and to compare alternative options for creating explanatory variables using dissimilarities between sequences. In section 2 we propose and discuss different approaches for measuring the relationship between a sequence and an out- 
come. In section 3 we present a study illustrating the performance of the approaches using different types of simulated sequence data. In section 4 we compare the same approaches using empirical data on work sequences, cumulative earnings, and poverty. Finally, in section 5 we report our conclusions on the discussed approaches.

\section{Methods}

In recent years, researchers in the social sciences have increasingly called attention to how existing methods understate the certainty with which individual cases are allocated to sequence clusters and overstate within-cluster homogeneity, arguing for the need for methodological developments (e.g., ?).

? and ? discuss the problems with linking SA cluster membership and a covariate. By assigning the same cluster membership value to all sequences in the same cluster, we are completely neglecting the possible within-cluster variation of the sequences. This is not a problem if the structure of the clustering is strong, i.e., there are clear subgroups in the data and we can be fairly certain of cluster memberships. Furthermore, the relationship between the sequences and the outcome of interest should be sufficiently explained by the cluster memberships (we refer to this as a "class-dependent outcome"). This refers to type A in Figure 1: there are two clear clusters and the value of the outcome - indicated by the shade of the dot - depends on the class only, not on the subject's position within the class (all within-class variation is random). A simple example of this situation would be having changes in the childhood family structure explaining educational outcomes in a case where parental separation would have the same kind of effect on all children. In this case, their position in relation to the clusters (e.g., due to the timing of the separation and possible parental repartnering) would not matter for explaining the sequence-outcome relationship.

In all other cases, however, the standard approach is potentially problematic. In a type B situation (Figure 1) the sequence-outcome link is similar to that of type A, but the clusters are overlapping. The weak clustering structure is a problem as it leads to misallocation 


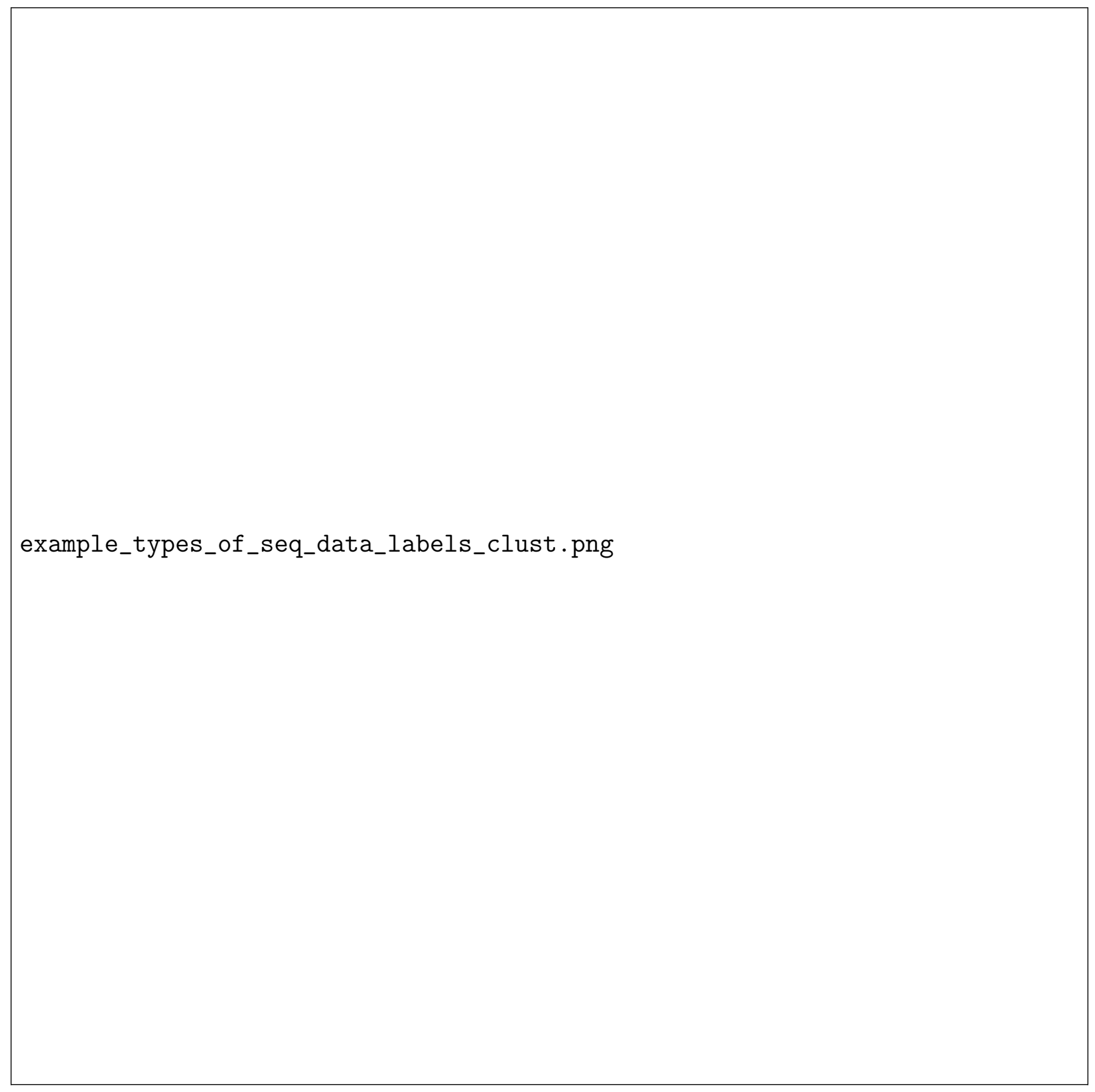

Figure 1: Illustration of four data types based on the strength of the clustering tendency and the type of the sequence-outcome link. The points refer to the relative positions of sequences in twodimensional space. The shade of the points refers to the value of an outcome variable. In panels $\mathrm{A}$ and B the value of the outcome depends on the class membership (classes differentiated by shape) and the within-class variation is random, while in panels $\mathrm{C}$ and $\mathrm{D}$ the value of the outcome depends on the relative positions of the sequences (here: increases along the vertical axis). The dashed lines show a partitioning suggested by a PAM clustering algorithm.

of sequences. Even if this misallocation is random, this can bias the estimates as in the analogous case of measurement error in covariates (cf. regression dilution/attenuation; e.g., 
?), and in some cases failing to account for this classification error can also lead to too small standard errors and p-values, increasing the risk of type I error (??).

In social sciences, we argue, it is often unrealistic to assume that any true underlying clusters exist (contrary to, e.g., pattern recognition applications). However, even if true clusters existed, they have been shown to be difficult to identify using existing methods (?) and thus the sequence-outcome link cannot be easily reduced to the relationship between fixed cluster memberships and an outcome. Typically, we have a situation where the the sequence typology derived from clustering can be regarded as an imperfect assignment of sequences to categories which approximate different ideal types. In this situation, the outcome depends on how strongly the sequences resemble the ideal types or how they relate to one another (their relative positions). Illustrations of such data with "similarity-based outcomes" are shown in panels $\mathrm{C}$ and $\mathrm{D}$ of Figure 1. A simplified example would be the relationship between employment trajectories and lifetime accumulated income. In such case, accounting for other factors such as education level, an individual 1 in a long stable employment career would have, on average, higher accumulated income than another individual 2 who never had a stable job. In such a situation the accumulated income individual 3 who entered the labour market at a later age and was consistently employed thereafter would be somewhere in between that of 1 and 2 (again, accounting for educational level). Careers more similar to individual 1 would tend to have higher incomes while careers more similar to individual 2 would tend to have lower incomes.

In a type $\mathrm{C}$ situation, we have a strong clustering structure from which we can easily name some representative or ideal-type sequences (such as normative school-to-work trajectories). In a type D situation, there is merely a weak clustering tendency or no clear structure at all, but different types of trajectories are nevertheless related to different levels of the outcome. In this situation, cluster analysis can be used as a tool for finding some representative sequences that help in assessing and interpreting the sequence-outcome relationship.

Few proposals to account for the uncertainty of the clustering result have been made so 
far. In a case for sequences being the outcome of interest, ? first brought up the idea of using so-called fuzzy or soft clustering methods for accounting for mixed cluster memberships of sequence data in a case where sequences were the outcome of interest. In terms of sequences as a predictor (the interest in this paper), to account for classification error, ? conducted robustness checks by excluding cases with poor silhouette values (reflecting a poor fit to their respective cluster; ?). In their study, excluding cases with low silhouette values led to relatively small deviations in estimates but a substantial loss of cases and a considerable increase in standard errors of the estimates.

In this section, we propose and discuss three alternatives to the traditional hard classification approach.

\subsection{Membership probability and representativeness}

If we assume to have fixed cluster memberships and class-dependent outcomes, our main goal is to assign individuals to their correct clusters. So-called fuzzy or soft clustering or soft classification is a form of clustering where individuals belong to clusters with a certain probability or degree. Instead of assigning subjects to one cluster and one cluster only - which can effortlessly be turned into an easily interpretable categorical variable - soft classification leads to a membership matrix, which is less straightforwardly usable in a regression model. ? proposed using the membership matrix as the outcome in a Dirichlet regression model, but to our knowledge no proposals on creating explanatory variables from a membership matrix of sequence data have been made so far. If we look beyond the SA literature, there have been some proposals in the latent class analysis (LCA) literature in creating independent variables from latent classes where - similarly to cluster analysis - true class memberships are unknown. The most interesting approach is the multiple pseudoclass method where individuals are randomly assigned into clusters multiple times based on their membership probabilities and the final estimates are obtained in a similar way to multiple imputation (???; for an application to sociological data, see ?). We apply a similar strategy in the 


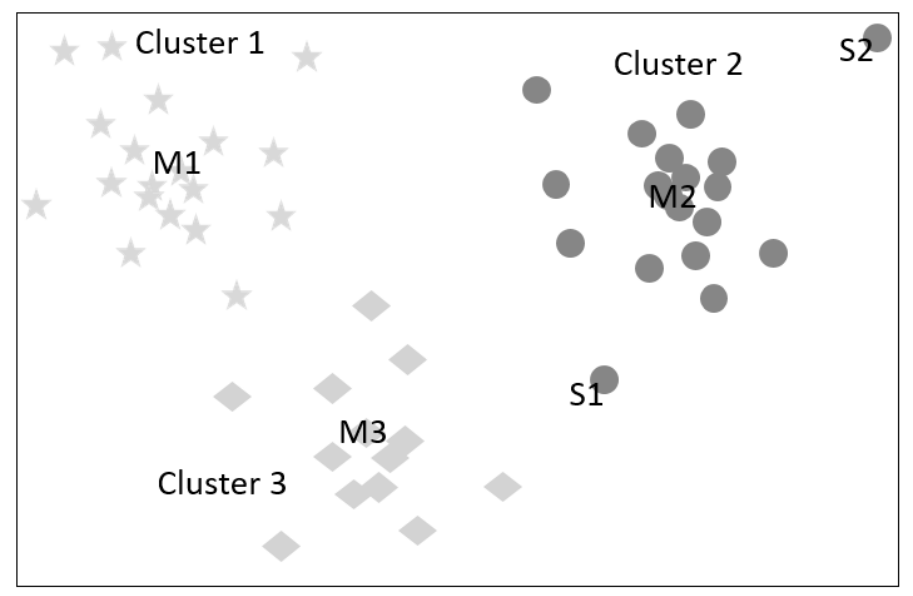

Figure 2: Example clusters with strong representatives (the medoids M1, M2, and M3) and two types of weak representatives (S1 and $\mathrm{S} 2$ ).

context of SA.

While likely an improvement over hard classification, soft classification and pseudoclass methods still assume that each subject belongs to a single cluster, but account for the uncertainty in the cluster assignments. We argue that this dependence on a single cluster is unrealistic in social sciences, as many individual characteristics are continuous in nature and there are an unlimited number of different life-courses instead of fixed categories.

If we do not believe in the existence of true clusters, but instead assume that the relative positions of the sequences matter more, we need to focus on their (dis)similarities directly. Since using pairwise dissimilarities in explaining an outcome as such is practically impossible, we turn our focus to the concept of representativeness.

Representativeness of sequences has been discussed in ? who consider different options including frequency, neighbourhood density, and centrality. Of these, centrality considers the distances or dissimilarities between sequences. Centrality can be calculated as the sum of dissimilarities between a subject and all (other) members in a group. The smaller the sum, the more central the subject; the most central subject is called the medoid. The closer a medoid is to a sequence or a group of sequences, the better representative it is to them.

As an example of how membership probability and representativeness differ, let us consider a situation visualised in Figure 2. Subjects M1, M2, and M3 are the medoids, i.e., the 
most central members of their respective clusters. As such they are the best single representatives to their clusters. At the same time, we can be fairly certain that they belong to their respective clusters; their membership probabilities are high regarding their own clusters and low regarding all other clusters.

Subjects S1 and S2, on the other hand, are both distant from the closest medoid M2, so they are much less representative to cluster 2 - and medoid M2 is much less representative of them than most of the other members. S1 and S2 are, however, different in their positioning. Subject S1 is of a mixed type - almost equally distant from medoids M2 and M3. Its membership probabilities for clusters 2 and 3 are thus similar, close to 0.5. Subject S2, however, is simply a distant subject: it is distant from medoid M2 but even further away from medoids M1 and M3. Even though it does not fit any cluster particularly well, its membership probability to cluster 2 is high, corresponding to strong certainty of being a member of cluster 2. Hence, it is easy to see that the membership probability itself is not always a good measure of representativeness.

If we are dealing with a type A or type B situation (class-dependent sequence-outcome relationship), the relative position within the cluster and thus subjects' representativeness is not an issue - unless we assume to find subjects that are not members of any clusters (outliers). However, in situations of type $\mathrm{C}$ and $\mathrm{D}$ representativeness is arguably more important and often theoretically more justified approach as we must consider subjects' positions in relation to others, e.g., by comparing them to some theoretical ideal types or medoids.

\subsection{Creating variables from sequences}

Table 1 presents different ways of constructing variables from sequences, two of which are based on a crisp clustering algorithm (in this case, the PAM algorithm; partitioning around medoids) and two based on a fuzzy clustering algorithm, here the FANNY algorithm (?).

Let $K$ be the number of clusters obtained from a clustering algorithm. We refer to the tra- 
Table 1: Variable construction for the simulation and empirical studies including two methods for crisp clustering (using the PAM algorithm; partitioning around medoids) and two for fuzzy clustering (using the FANNY algorithm).

\begin{tabular}{llll}
\hline Name & Clustering method & Variable construction & Variable type \\
\hline Hard classification & Crisp (PAM) & Cluster membership & Dummies \\
Soft classification & Fuzzy (FANNY) & Membership degree & Continuous \\
Pseudoclass & Fuzzy (FANNY) & Multiple pseudoclass technique & Dummies \\
Representativeness & Crisp (PAM) & (Modified) distance to medoids & Continuous \\
\hline
\end{tabular}

ditional approach of constructing a categorical variable with $K$ categories from crisp cluster memberships as hard classification. Soft classification refers to using membership probabilities from fuzzy clustering as $K$ continuous variables (which sum to 1 for each subject). In both types of variables, one cluster is typically chosen as a reference and the respective (dummy or probability) variable is omitted from the model.

Pseudoclass is the equivalent of the multiple pseudoclass technique in probabilistic LCA, where we draw multiple samples of cluster memberships from fuzzy clustering and for each sample estimate a model with a categorical membership variable the usual way. Finally, we combine the results across the models similarly to the multiple imputation technique (?). This type of an approach is fairly common in the LCA literature despite some more recent studies (e.g., ?) showing that this approach might not provide improvements over hard classification.

Finally, we construct a variable that takes into account representativeness. For this technique we need to define a set of representative sequences which we can choose, e.g., based on theory or from using a clustering algorithm. Here we use a crisp clustering algorithm for finding medoids and calculate the dissimilarity of each sequence to each cluster medoid based on the same distance matrix as for the hard clustering (PAM). We transform these dissimilarities to representativeness values so that value 1 refers to perfect representation and 0 to poorest representation. More specifically, we define the representativeness value of 


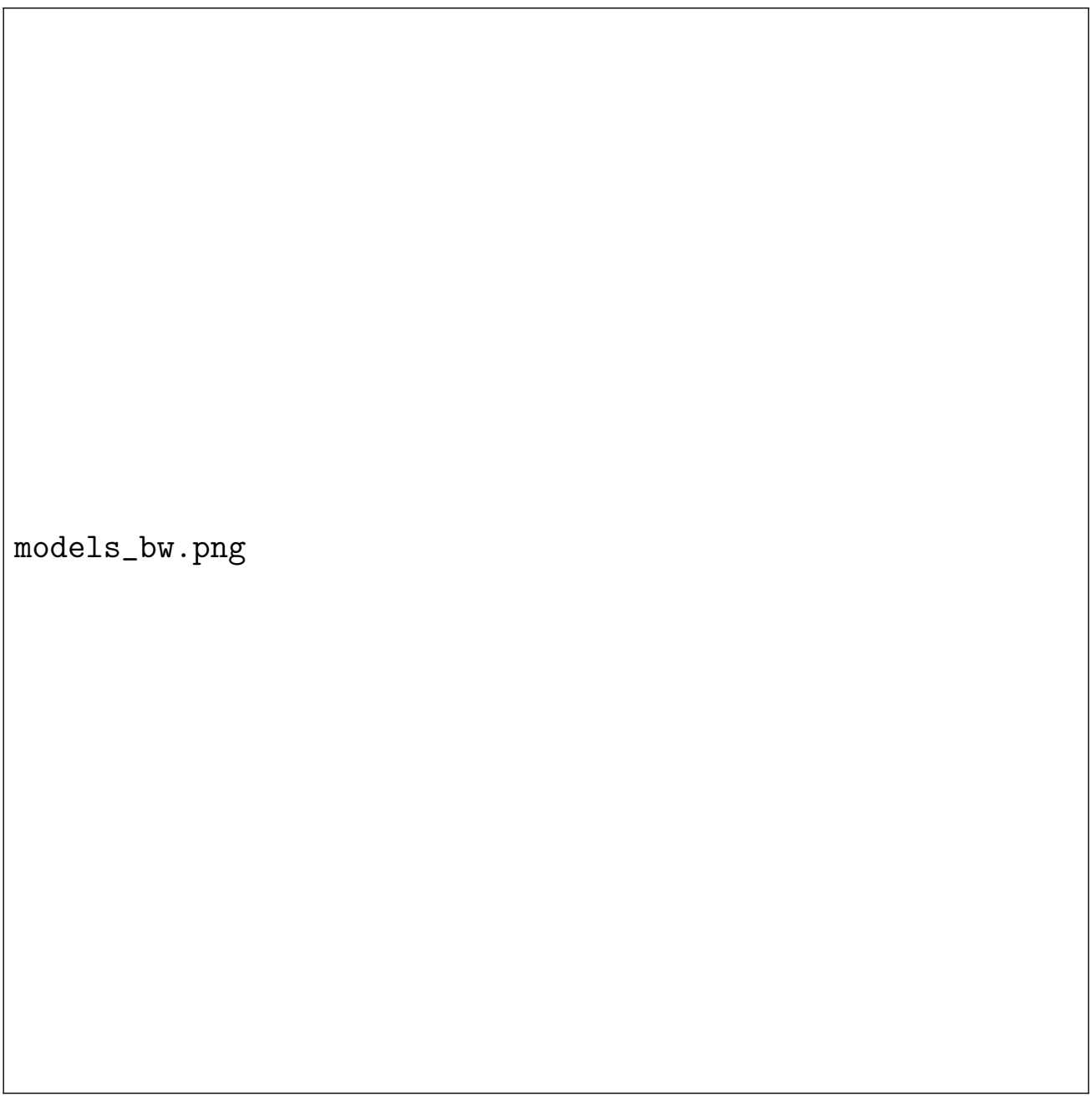

Figure 3: Clusters of sequences simulated from three types of mixture Markov models with varying clustering tendencies (weak, reasonable, and strong). The shades refer to four simulated states.

representative $k$ (here: the medoid of cluster $k$ ) to sequence $i$ as

$$
R_{i}^{k}=1-\frac{\text { distance of sequence } i \text { to representative } k}{\text { maximum distance between two sequences }}
$$

This leads to $K$ continuous variables for subsequent analysis (which do not sum to 1 ).

\section{$3 \quad$ Simulation study}

In this section we illustrate how different approaches work for predicting the outcome when the sequence-outcome relationship is class-dependent or similarity-based. All analyses were done in the $\mathrm{R}$ environment (?), using packages cluster (?), seqHMM (?), TraMineR 
(?), ggplot2 (?), and dplyr (?). The codes to reproduce the simulation experiment with and additional analyses can be found on Github (https://anonymous.4open.science/r/ seqs2vars-A223).

We first generated sequence data by creating three mixture Markov models (MMMs) with varying clustering tendencies, each with four states and four mixture components ("clusters"). We simulated 10,000 sequences of length 20 from each of these models. We then calculated dissimilarities using Optimal Matching for spell sequences (OMspell) with constant substitution costs (?). This measure was chosen, as it is sensitive to sequencing and as such well suited for analysing data generated with a Markovian model. We then clustered the sequences using PAM and FANNY. Assessed using the average silhouette width (based on PAM) as a measure of clustering tendency (ASW; ?), the first model generated sequences with strong clustering tendency (ASW being around 0.8), the second generated sequences with a reasonable clustering tendency with some overlap between sequences from different submodels (ASW around 0.6), and the third generated sequences with a weak clustering tendency (ASW around 0.3). Figure 3 shows samples of clustered sequences. Using the clustering solutions and the corresponding dissimilarity matrix, we then created several covariate matrices $X$ based on the methods outlined in Section 2.2 and summarized in Table 1. For each generated matrix $X$ we then generated a response variable $y$ by

$$
y_{i}=x_{i} \beta+\epsilon_{i}, \quad \epsilon \sim N\left(0, \sigma^{2}\right),
$$

with $\beta=(0,1,1,-1)^{\prime}$ and $\sigma=0.25$ for each $X$.

Using each of these datasets, we ran Monte Carlo simulation where for each replication we sampled 1,000 of the original sequences and a corresponding $y$ from the full dataset and computed covariate matrix $X$ using all of the methods presented in Table1. For FANNY, we fixed the membership exponent to 1.4, as larger values often led to complete fuzzyness and convergence issues during the simulations. We then split the data in half and used the first 
half to estimate the regression model $y_{i}=x_{i} \beta+\epsilon_{i}$. Finally, we used this model to predict the values of the hold-out sample and computed the corresponding root mean squared error (RMSE) of the predictions. This allowed us to estimate the out-of-sample performance of our methods, also taking into account the potential bias and uncertainty stemming from the construction of $X$ using the subsamples instead of the full population data.

In reality, sequence data is unlikely to have been generated by such simple Markovian models that we used, and the relationship between sequences and outcome variables is more complex. Thus, the following results reflect more of a best case scenario, and in practice the differences between the methods and potential errors could be much larger than observed here.

Figure 4 shows the average RMSE and 95\% percentile intervals from 10,000 replications for different data-generating models and estimation methods. We see that for classificationbased data the prediction improved (RMSE decreased) when the clustering tendency strengthened. Not surprisingly, the estimation based on hard classification performed best with strong, clear clusters, while soft classification performed, on average, slightly better in the cases where we had classification error (data with a reasonable or weak clustering tendency). The hard classification method produced widest percentile intervals - its performance was the most inconsistent. When the outcome was generated based on membership probabilities, the clustering tendency did not have a strong impact on the average RMSE when using the estimation method that matched the data generation process (soft clustering, the best case scenario), whereas other methods performed best with stronger clustering tendency. On the other hand, when the data was generated based on representativeness (the case we argue is typically the most realistic in social sciences), the clustering tendency did not have a clear impact on the average RMSE for any of the methods, and all methods produced results not far from the theoretical value of 0.25 (the standard deviation of the noise term $\epsilon$ ). Still, the predictions based on representativeness outperformed others, most notably the commonly used hard classification approach. The pseudoclass method, inspired by a similar approach 
from the LCA literature, did not perform particularly well in any setting. The soft classification was the most robust method over all scenarios, and naturally performed best in the middle panel with a matching data generating process.

We also performed additional experiments where the original data generation and covariate creation was done with FANNY based hard classification and gravity centers, a potential alternative to our representativeness measure (?). We also tested ranking the methods based on BIC instead of RMSE (excluding the pseudoclass method for which BIC is not defined). These results are available in the supplementary material on Github. These additional simulations were in line with the conclusions of these main results, with FANNY-based hard classification performing similarly to the PAM-based hard classification and the gravity center method being similar to the representativeness method.

\section{Empirical study}

In this section we illustrate the performance of the four methods with an empirical research problem: predicting a continuous earnings variable or a binary poverty variable with simple two-state sequences of employment trajectories.

The timing, length, and frequency of employment and unemployment spells have a profound effect on earnings (??). These features of one's occupational career determine the opportunities for on-the-job human capital accumulation, while also signalling a worker's competence and unobservable qualities to potential employers (?). Over time, the cumulative effects on earnings can be substantial (?).

The data used in this example come from the Swedish population registers. The dataset comprises a sample of all residents of Sweden who turned 18 years old in 1997 and who lived continuously in the country until $2017(N=10,000)$. In other words, we observe all subjects from age 18 to age 38 . Yearly states are coded as "1 Working" and "2 Not working" based on income and employment information from the Longitudinal Integrated Database for Health Insurance and Labour Market Studies (LISA). Individuals who declared any income 


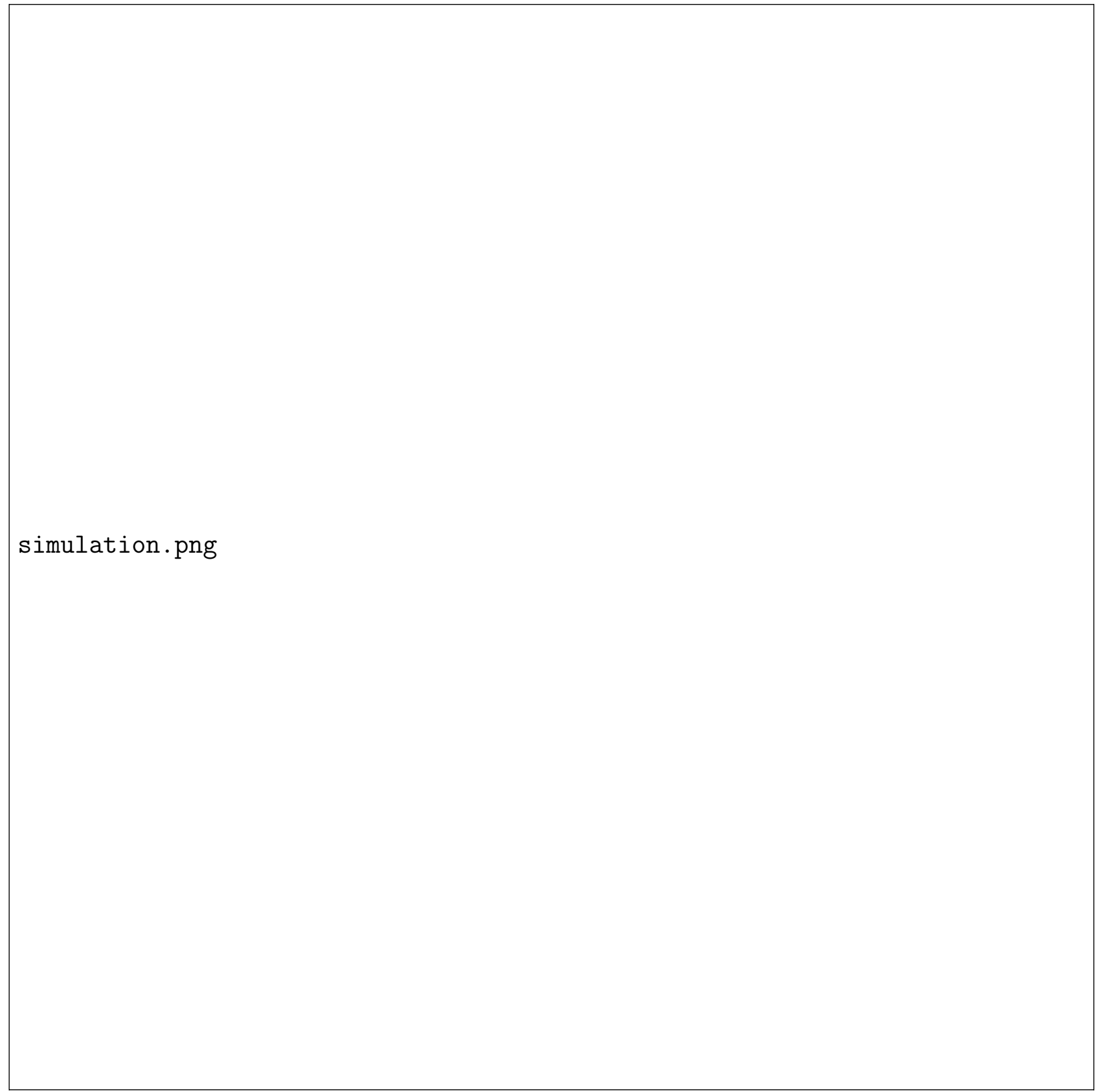

Figure 4: Average root mean square errors of predictions from 10,000 simulations with $95 \%$ percentile intervals. 
from employment in a given year are coded as employed. Other variables included in the multivariate analysis are retrieved from the Total Population Register (RTB).

We were interested in two outcome variables: (1) the probability of being in the lowest income quintile at the end of the sequence (a measure of poverty) and (2) the square root of cumulative income over the entire sequence (in 1000 SEK). Income in this case was income from wages, business, and other economic activity, including social benefits related to economic activity (such as parental leave and sick leave compensations). We also had measurements of characteristics of the individual and of their family background at the start of the sequence. These were: region of residence (Metropolitan areas, smaller cities, countryside), mother's education, father's education, mother's employment status, father's employment status, and sex.

The models for poverty were estimated using logistic regression and the models for income used ordinary least squares regression using four different methods to predict the outcome with employment histories. In both cases, we controlled for characteristics of the individual and of their family at the start of the sequence.

For the clustering of sequences, we used a dissimilarity measure that is sensitive to the duration of (un)employment spells, namely Optimal Matching with a substitution cost of 2 and an indel cost of $1(?)$. We chose a solution with five clusters for our example, with clusters differing in timing, prevalence, and continuity of employment. Figure 5 shows the medoids and the index plots for the sequences within each cluster of the hard classification (PAM) solution. The first cluster, Mainly no Work, is characterised by the prevalence of spells without work, being the cluster with longer and more prevalent states outside of employment. The second cluster, Unstable Work is characterised by frequent transitions in and out of work, which become more common towards the end of the observation period. The cluster Late Entry is characterised by very late entry into the labour market, mostly after age 28, and often followed by frequent transitions in and out of work. Delayed Entry is characterised by spells of unemployment or inactivity in the first seven years (i.e. between 
ages 18 and 25) and by a transition to mostly stable employment afterward. Last, Early Entry is characterized by a long spell in employment, starting early in the observation window. A classification assigning cluster membership based on the highest membership probability obtained by the FANNY algorithm showed similar qualitative patterns, with the majority of all sequences in each of the five classifications being allocated to the same cluster. There were, however, minor differences in allocation. First, the lowest degree of overlap between the two classifications was $57 \%$ for the category Unstable Work, which had $35.8 \%$ of its sequences allocated to Late Entry in the soft classification. Second, 33.7\% and $20.2 \%$ of the Early Entry and Delayed Entry, respectively, were allocated to Unstable Work. These differences result in a higher share of the Working state at later positions in the cluster Unstable Work of the soft classification than in the hard classification, but otherwise remarkably similar patterns in the other clusters (see tables A.2 and A.3, as well as figure 7).

Earlier research suggests that the varying degrees of attachment to employment and the different lengths of employment spells found in each cluster would have distinct outcomes in terms of poverty and cumulative earnings. This can be studied using the cluster variable as a predictor of these two outcomes, in a similar way to the study by ?. In addition, we repeated the analysis using the other approaches described in the simulation study in section 3. namely pseudoclass, soft classification, and representativeness.

In this case we did not believe that any true employment clusters exist or that the outcomes would be class-dependent. Instead, we assumed that the relationship between the work trajectory and the outcome (income or poverty) is similarity-based and expected that representativeness would be the most appropriate measure to use.

Our analysis highlights the substantial differences in how the different types of sequence variables perform as predictors. Before showing the full results, we illustrate the differences in predicted values with a simple example.

When using hard cluster memberships and setting Early Entry (cluster 5) as the reference 
sequences.png

Figure 5: Yearly employment state distribution and medoids for 5-cluster PAM solution. Sweden, random sample of the cohort entering working age in 1998. $\mathrm{N}=10000$. 
category, the expected cumulative income for an individual would be:

$$
\mathbb{E}\left(I_{i}\right)=\beta_{0}+\beta_{1} C_{i}^{1}+\beta_{2} C_{i}^{2}+\beta_{3} C_{i}^{3}+\beta_{4} C_{i}^{4}+Z_{i} \gamma
$$

where $\mathbb{E}\left(I_{i}\right)$ is the expected (square root of) cumulative income for individual $i, \beta_{0}$ the intercept, $\beta_{k}$ the regression coefficient, $C_{i}^{k}$ membership in cluster $k$ of sequence $i$ (0 or 1 for hard classification), and $Z_{i}$ represents all other covariates in the model and $\gamma$ the vector of their coefficients. Now, consider three sequences from the Mainly no Work cluster (Cluster 1), (M), (A), and (B) consisting of employment (E) and unemployment/inactivity (U):

(M) U-U-U-E-U-U-U-U-U-U-U-U-U-U-E-U-E-E-U-U

\section{(A) $\mathrm{U}-\mathrm{U}-\mathrm{U}-\mathrm{U}-\mathrm{U}-\mathrm{U}-\mathrm{U}-\mathrm{U}-\mathrm{U}-\mathrm{U}-\mathrm{U}-\mathrm{U}-\mathrm{U}-\mathrm{U}-\mathrm{U}-\mathrm{U}-\mathrm{U}-\mathrm{U}-\mathrm{U}-\mathrm{U}$}

\section{(B) $\mathrm{E}-\mathrm{E}-\mathrm{E}-\mathrm{E}-\mathrm{E}-\mathrm{U}-\mathrm{E}-\mathrm{E}-\mathrm{E}-\mathrm{U}-\mathrm{U}-\mathrm{U}-\mathrm{U}-\mathrm{U}-\mathrm{U}-\mathrm{U}-\mathrm{U}-\mathrm{U}-\mathrm{E}-\mathrm{U}$}

A hard-classification method (PAM) assigns these three sequences to the same cluster, which is characterised by long unemployment spells. Here, sequence (M) is the medoid of the cluster and shows a pattern of mostly unemployment, (A) consists solely of unemployment spells, and (B) is an outlier with a long spell of nearly continuous employment that ends

halfway through the period. For the case of hard cluster memberships as predictors, the square root of expected 20-year cumulative earnings for all these sequences is reduced to:

$$
\mathbb{E}\left(I_{i}\right)=\beta_{0}+\beta_{1}+Z_{i} \gamma=64.69-33.53+Z_{i} \gamma
$$

Note that cluster membership is reflected in the equation as a single parameter referring to the cluster assigned to all three sequences (in this case the first cluster).

For the sake of simplicity, if we assume that the individuals in question belonged to the baseline category for all other covariates, the predicted value of the square root of the 20-year cumulative earnings (in thousands of SEK) is 31.16, which translates approximately to SEK 970,000 for the three cases of (M), (A) and (B). 
Likewise, for the pseudoclass approach, the equation is:

$$
\mathbb{E}\left(I_{i}\right)=\beta_{0}^{*}+\beta_{1}^{*}+Z_{i} \gamma^{*}=63.28-27.49+Z_{i} \gamma^{*},
$$

where the coefficients are averages over multiple pseudoclass samples (the estimates are different compared to those from the hard classification method, as reflected by the asterisks). The equivalent square root of predicted earnings is 35.79 , translating into approximately SEK 1,280,000 for all of (M), (A) and (B).

As discussed earlier, a key difference between hard clustering and pseudoclass is that pseudoclass assigns cluster memberships based on the estimated membership probabilities from a fuzzy cluster solution. The coefficients represent the averaged cluster membership effect over all the replications and the standard errors are adjusted to reflect the uncertainty deriving from the probabilistic cluster allocation. In this way, pseudoclass deals with the problem of treating group assignment as certain by adjusting the estimated parameters and standard errors so that they reflect the uncertainty in cluster allocation. Yet, pseudoclass is similar to hard classification in that it attributes an uniform effect to all members of the same cluster, as our example shows. Also note the difference in the estimates between the methods: pseudoclass tends to shrink estimates toward the average (??), which makes it the most conservative of all the methods in terms of finding differences between the groups.

In contrast, the equations for the soft classification and representativeness methods reflect within-cluster variability by incorporating more parameters and changing the predictors into continuous measures. For soft classification, the equation includes $k-1$ parameters representing membership probabilities, leading to

$$
\mathbb{E}\left(I_{i}\right)=69.31-56.31 P_{i}^{1}-12.36 P_{i}^{2}-19.67 P_{i}^{3}+9.39 P_{i}^{4}+Z_{i} \gamma^{\dagger},
$$

where $P_{i}^{k}$ is the probability that the sequence $i$ belongs to the soft cluster $k$ (similarly to the hard cluster approach, one of the cluster membership probabilities is left out of the equation 
because the probabilities sum to 1). Instead of sampling-based adjustment to uncertainty in the pseudoclass method, here the uncertainty of classification is incorporated into the covariates $P_{i}^{k}$ themselves. Imputing the membership probabilities for each sequence yields:

$$
\begin{aligned}
& \mathbb{E}\left(I_{M}\right)=69.31-56.31 \times 0.83-12.36 \times 0.03-19.67 \times 0.08+9.39 \times 0.05+Z_{i} \gamma^{\dagger} \\
& \mathbb{E}\left(I_{A}\right)=69.31-56.31 \times 0.86-12.36 \times 0.03-19.67 \times 0.05+9.39 \times 0.04+Z_{i} \gamma^{\dagger} \\
& \mathbb{E}\left(I_{B}\right)=69.31-56.31 \times 0.43-12.36 \times 0.16-19.67 \times 0.20+9.39 \times 0.13+Z_{i} \gamma^{\dagger}
\end{aligned}
$$

For (M), the predicted value of square root earnings (in $1000 \mathrm{SEK}$ ) is 21.01, translating into about SEK 445,000, while for (A) the same predicted value is 19.9, translating into about SEK 396,000, and for (B) the value is 40.42, which translates into about SEK 1,632,000. Thus, the estimation based on soft classification captures the considerable earnings difference that results from the differences in the presence of unemployment spells within the three sequences.

In a similar vein, the equation using representativeness incorporates multiple parameters (which do not have to sum to 1 ):

$$
\mathbb{E}\left(I_{i}\right)=-4.58-1.12 R_{i}^{1}-11.68 R_{i}^{2}+26.41 R_{i}^{3}+25.70 R_{i}^{4}+48.25 R_{i}^{5}+Z_{i} \gamma^{\dagger}
$$

where $R_{i}^{k}$ is the representativeness value of the representative sequence $k$ (here: the medoid of cluster $k$ ) to sequence $i$. The resulting equations from imputing the representativeness values of each example sequence are:

$$
\begin{aligned}
& \mathbb{E}\left(I_{M}\right)=-4.58-1.12 \times 1.00-11.68 \times 0.45+26.41 \times 0.60+25.70 \times 0.50+48.25 \times 0.25+Z_{i} \gamma^{\dagger}, \\
& \mathbb{E}\left(I_{A}\right)=-4.58-1.12 \times 0.80-11.68 \times 0.25+26.41 \times 0.40+25.70 \times 0.30+48.25 \times 0.05+Z_{i} \gamma^{\dagger}, \\
& \mathbb{E}\left(I_{B}\right)=-4.58-1.12 \times 0.65-11.68 \times 0.60+26.41 \times 0.50+25.70 \times 0.45+48.25 \times+.45+Z_{i} \gamma^{\dagger},
\end{aligned}
$$


The predicted square root earnings (in $1000 \mathrm{SEK}$ ) for sequence (M) using the representativeness method is 29.80, translating into approximately SEK 888,000. For sequence (A) it is 12.29 which translates to approximately SEK 151,000. Last, for sequence (B) the value is 34.17 , translating into about SEK 1,167,000. As in the case of soft-classification, representativeness also captures the differences in earnings between the three sequences even when the clustering algorithm has assigned them to the same group.

As illustrated by the previous chapters, the four approaches differ in terms of how predictions are calculated, which also means that they differ in terms of interpreting the estimated modelling results. Interpretation is most straightforward for hard clustering, as it is interpreted as any categorical variable: parameter coefficients $\beta_{k}$ correspond to the average differences between members of cluster $k$ and the members of the reference category. The estimates from pseudoclass, even though calculated in a different manner, are interpreted in the same way. For soft classification, the coefficients are interpreted as the difference in the outcome (or the probability of the outcome, in terms of the logistic model) between a fully certain member of cluster $k$ (whose membership probability is 1 for that cluster and 0 for others) and a fully certain member of the reference category. For representativeness, the parameter estimates are difficult to interpret as such, as they correspond to dissimilarities/distances to all of the medoids (or other representative sequences). When interpreting the parameter estimates, one cannot simply interpret one of them but must consider all at the same time. However, average marginal effects (AMEs) or average marginal predictions (AMPs) can be used for easier interpretation and they are also comparable across all four approaches.

Here we show model results as AMPs for all four approaches. AMPs and AMEs are similar concepts, except that instead of comparisons to a reference case as in the more typical AMEs, the AMPs, also known as average adjusted predictions show marginal predictions under some interesting configurations, in our case, at the medoids obtained from the hard classification. Specifically, separately for each medoid, we predicted the outcome for each 
individual by replacing their observed representativeness values with the representativeness values of the medoid (while keeping other covariates at their observed values) and then calculated the average of the predictions over all individuals. Similarly, for soft classification, we replaced the observed membership probabilities of each individual with those of the medoids, and with hard classification, AMPs are calculated by replacing the observed cluster memberships. Finally, the pseudoclass AMPs are calculated for each pseudoclass replication as with hard classification, and the set of AMPs obtained from all pseudoclass replications are then combined using Rubin's rules.

The top two panels (a and b) in Figure6 display the average marginal predictions for the clusters (hard and soft classification and pseudoclass) or medoids of each cluster (representativeness) by outcome and estimation approach. The estimates largely agree with each other, predicting worse outcomes for the Mainly no Work cluster concerning both discrete (poverty) and continuous (income) outcomes. Overall, representativeness- and soft classification-based estimates are more precise, with narrower confidence intervals. This is particularly evident for the discrete outcome. One exception to this pattern is the representativeness effect for Unstable Work, where representativeness is the least precise of all estimation methods. Last, it is evident that in most cases pseudoclass is the least precise of all methods, often displaying the widest confidence intervals.

Even though the different methods mainly agree with each other, they would, however, lead to qualitatively different conclusions regarding the sequence-outcome relationship. For example, when considering cumulative income, the estimates from hard classification would typically be interpreted as showing no statistically significant differences between four out of five clusters (the only differing one being the Mainly no Work cluster). Representativeness and soft classification, however, show that Unstable Work is also connected to lower cumulative earnings than Delayed Entry and Early Entry. Furthermore, representativeness and to some extent also soft classification suggest that Unstable Work is related to a higher risk of poverty than Early Entry, Delayed Entry, and Late Entry. 


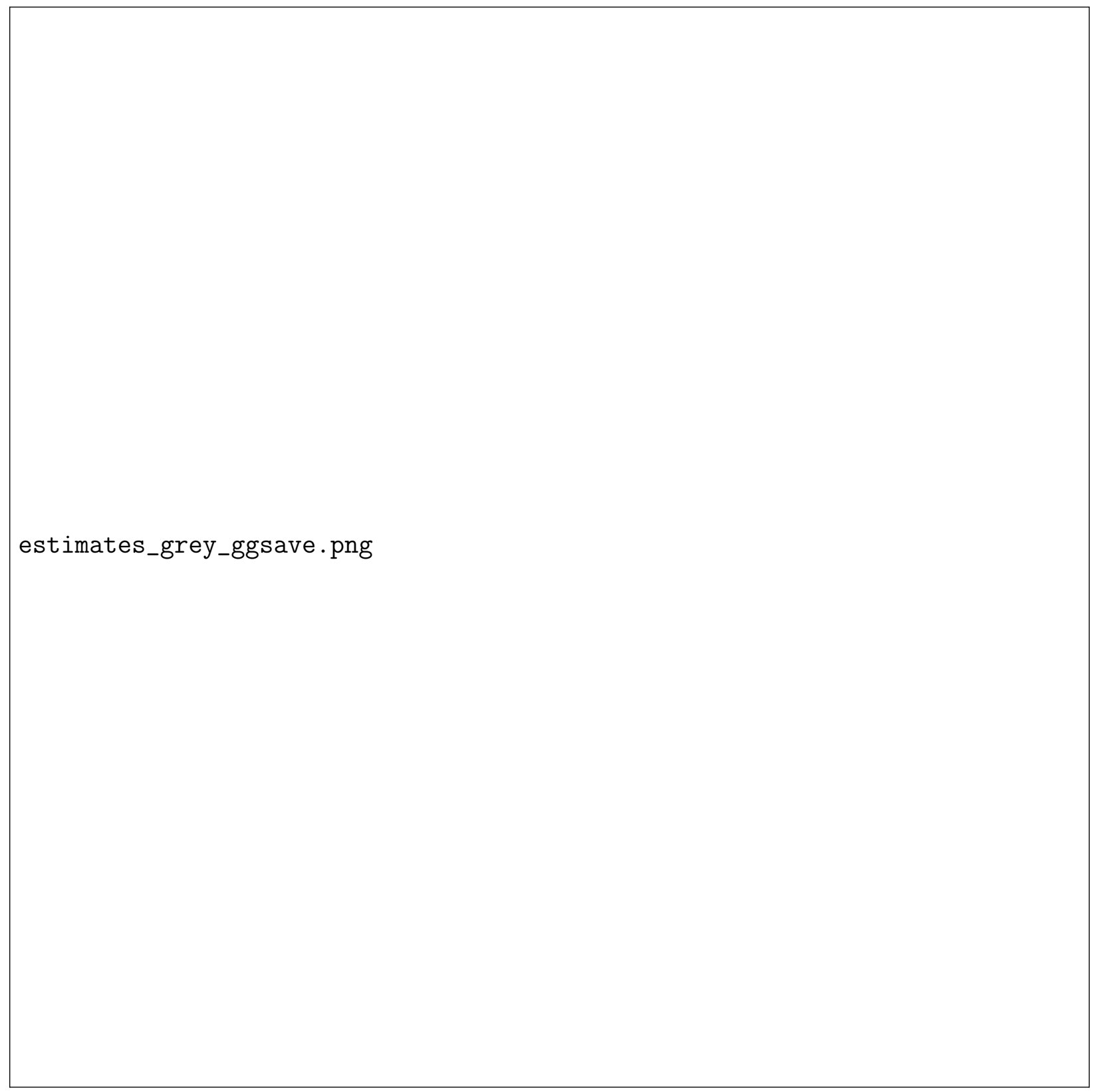

Figure 6: Average marginal predictions, root mean squared errors, and Brier scores by estimation method and outcome. 
The lower panels (c and d) in Figure 6 show the root mean square errors (RMSE) and Brier scores which we use to assess the accuracy of the predictions. We computed them using a leave-one-out cross-validation method over 100 folds and estimated confidence intervals by using bootstrapping with 1000 replications. As expected, representativeness produced more accurate estimates in both cases than the hard classification and pseudoclass methods, while soft classification was relatively close to the performance of representativeness, especially in the continuous case.

See the appendix for further results for the empirical study such as descriptive statistics and parameter estimates from each model.

\section{Discussion}

In this paper it was our aim to bring forward and discuss the problems of the traditional approach of creating variables from SA clusters and to propose some alternative approaches.

Our simulation study demonstrated how the type of the data generating process affects the performance of the different methods. In cases with true but unknown clusters, hard classification worked well on data with strong clustering tendency while soft classification was more consistently better on data with weaker clustering tendencies (i.e., when classification error is an issue). However, when there were no true clusters to begin with but the sequence-outcome relationship was assumed to be similarity-based, in our simulations representativeness clearly outperformed other methods.

We also studied the performance of the methods on empirical data, where we predicted two types of income-related variables (a continuous cumulative income variable and a binary poverty measure) with simple employment trajectories and control variables. In this case we assumed that the relationship between the sequences and the outcome would be closest to the similarity-based setup and expected that the representativeness measure would result in better predictions than the other methods. This was indeed confirmed by our analyses using cross-validation, but the advantage of using representativeness was not as evident in 
the empirical case as it was in the simulations. Soft classification was almost equally good for the continuous outcome, but performed less well when the outcome was binary.

We argue that typically in social sciences subjects are more or less hybrids of multiple ideal types and the outcome variable of interest is also affected by multiple factors with varying magnitudes, which is not properly captured by hard classification into clusters.

In earlier LCA literature, the pseudoclass method has been hypothesised to be better in accounting for the uncertainty due to clustering. The benefit of pseudoclass method over other proposed alternatives is that it tries to adjust for the uncertainty in the classification without altering the interpretation of the model in terms of the corresponding predictors. However, in our simulation and empirical studies its performance was less than convincing, which is in line with recent LCA literature (??). The pseudoclass method is also computationally the most demanding of the considered methods. While our pseudoclass approach is not based on latent class models but fuzzy clustering of sequence dissimilarities, based on all these findings we cannot recommend the pseudoclass method as an alternative to the traditional hard classification technique.

Soft classification with mixed memberships account for uncertainty of membership allocation and as such it is a clear improvement over the traditional hard classification with fixed memberships. The potential problem with soft classification is its inability to deal with cases that are not well represented by any of the ideal types (outliers). Similarity-based approaches such as representativeness take into account the closeness of the sequence to the ideal types while also distinguishing between mixed types and outliers. Other similarity-based measures, such as measures based on multidimensional scaling may also work, especially when the data show clear and easily interpretable principal components or when the goal is to construct a control variable (where interpretation of the effects of the sequence variables is not relevant). If outliers are not a big issue, soft classification and representativeness measures are expected to lead to relatively similar results. In this case soft classification could be favoured due to simpler interpretation. 
While in theory, the use of representativeness and membership probabilities can induce some level of multicollinearity to the modelling, we do not see this as a major issue as multicollinearity affects only the interpretation of individual predictors, and in these cases, the effects of these sequence-related variables are best to be considered as a whole (as in our examples).

Related to multicollinearity, in this paper we fixed the number of clusters/medoids to be the same across the different approaches for comparability. In practice, it may be advisable to use a smaller number of clusters/medoids for representativeness and possibly also for soft classification in comparison to hard classification, because of the continuous nature of these measures. For example, in our simple empirical example, dissimilarities to sequences of "always working" and "never working" capture the same information (causing multicollinearity), so in practice adding only one of these as a representativeness predictor would be sufficient.

To conclude, in this paper we demonstrated the importance of considering how sequences and the outcome variable of interest are related, and the need to adjust the analysis accordingly. If true underlying clusters are expected to exist, then hard or soft classification methods should be preferred (depending on how big an issue classification error is expected to be). In social sciences, the whole idea of the existence of any "true clusters" is often implausible. Often the main purpose of cluster analysis is to reduce the complexity of the sequence data, in which case similarity-based approaches or soft classification should be considered. Based on our analyses, the representativeness method shows promising results, and perhaps in future also other alternatives emerge. We hope this work will encourage further discussion and research on combining sequence analysis and subsequent modelling. 


\section{A Appendix: Empirical study}

Table A.1: Outcome variables by cluster

\begin{tabular}{lccc}
\hline Cumulative income over 10 years & & & \\
\hline Cluster & Mean & SD & $\mathrm{N}$ \\
\hline Mainly no work & 1520.71 & 1502.40 & 1019 \\
Unstable work & 3076.23 & 1186.19 & 1089 \\
Late entry & 3606.48 & 1544.39 & 1426 \\
Delayed entry & 4503.90 & 1894.54 & 2703 \\
Early entry & 4632.64 & 1420.23 & 3763 \\
\hline \% in Poverty at year 10 & & \\
\hline Cluster & $\%$ & Count & $\mathrm{N}$ \\
\hline Mainly no work & $61.14 \%$ & 623 & 1019 \\
Unstable work & $28.10 \%$ & 306 & 1089 \\
Late entry & $11.64 \%$ & 166 & 1426 \\
Delayed entry & $7.73 \%$ & 209 & 2703 \\
Early entry & $8.64 \%$ & 325 & 3763 \\
\hline
\end{tabular}

Table A.2: Cross-tabulation between cluster assignments by hard classification (PAM) and soft classification (based on the maximum membership probabilities from FANNY).

\begin{tabular}{rlllll}
\hline & \multicolumn{5}{c}{ Soft-classification } \\
Hard Classification & Early entry & Delayed entry & Unstable work & Mainly no work & Late entry \\
\hline Early entry & $66.3 \%$ & $0.0 \%$ & $33.7 \%$ & $0.0 \%$ & $0.0 \%$ \\
& $(2494)$ & $(0)$ & $(1269)$ & $(0)$ & $(0)$ \\
Delayed entry & $0.0 \%$ & $65.4 \%$ & $20.2 \%$ & $0.1 \%$ & $14.2 \%$ \\
& $(0)$ & $(1769)$ & $(546)$ & $(3)$ & $(385)$ \\
Unstable work & $0.2 \%$ & $0.0 \%$ & $57.0 \%$ & $7.0 \%$ & $35.8 \%$ \\
& $(2)$ & $(0)$ & $(621)$ & $(76)$ & $(390)$ \\
Mainly no work & $0.0 \%$ & $0.0 \%$ & $0.0 \%$ & $91.9 \%$ & $8.1 \%$ \\
& $(0)$ & $(0)$ & $(0)$ & $(936)$ & $(83)$ \\
Late entry & $0.0 \%$ & $15.1 \%$ & $0.6 \%$ & $1.5 \%$ & $82.8 \%$ \\
& $(0)$ & $(215)$ & $(9)$ & $(21)$ & $(1181)$ \\
\hline
\end{tabular}


Table A.3: Average soft classification probabilities by hard classification cluster

\begin{tabular}{rlllll}
\hline & & \multicolumn{5}{c}{ Soft-classification } \\
Hard Classification & Early entry & Delayed entry & Unstable work & Mainly no work & Late entry \\
\hline Early entry & $63.9 \%$ & $7.0 \%$ & $22.3 \%$ & $0.9 \%$ & $5.9 \%$ \\
Delayed entry & $5.3 \%$ & $43.5 \%$ & $23.7 \%$ & $3.0 \%$ & $24.5 \%$ \\
Unstable work & $12.3 \%$ & $18.6 \%$ & $32.4 \%$ & $10.3 \%$ & $26.5 \%$ \\
Mainly no work & $2.7 \%$ & $9.6 \%$ & $7.1 \%$ & $64.5 \%$ & $16.1 \%$ \\
Late entry & $3.9 \%$ & $30.9 \%$ & $14.2 \%$ & $9.9 \%$ & $41.1 \%$ \\
\hline
\end{tabular}


hard_cluster.png

(a) Hard classification

fuzzy_cluster_weighted.png 


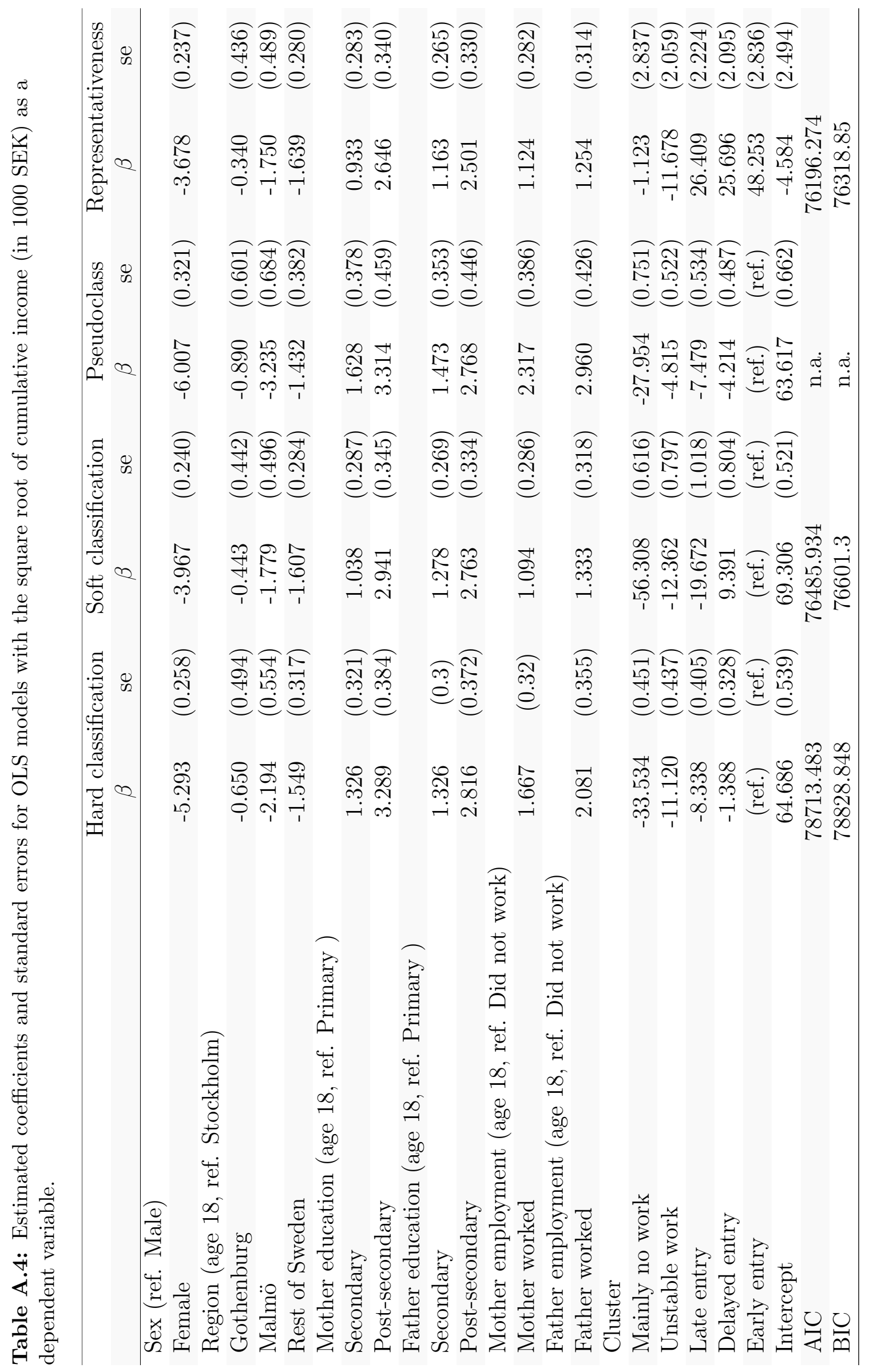




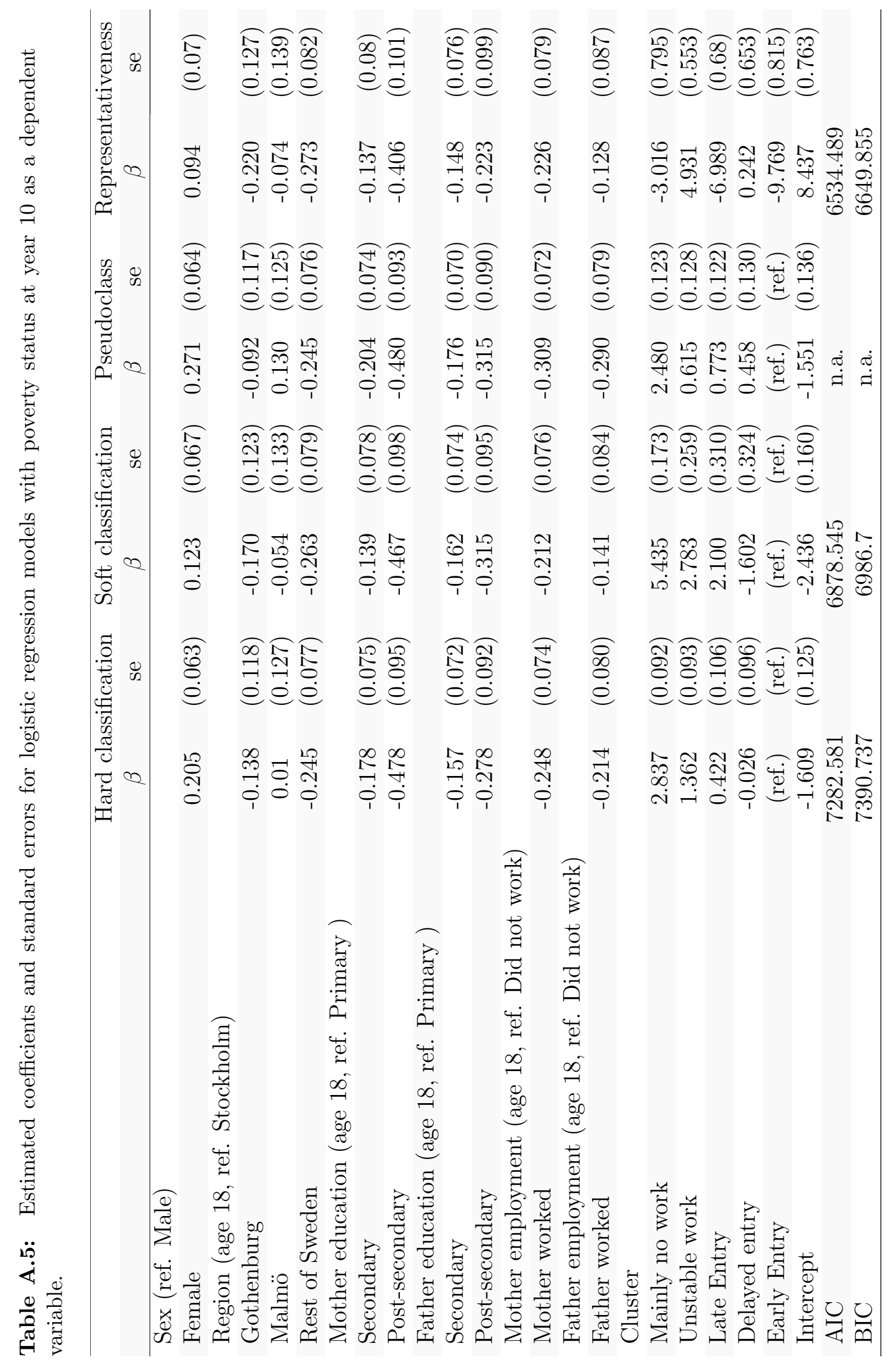


Table A.6: Correlation coefficients between soft classification probabilities

\begin{tabular}{rrrrrr}
\hline & Early entry & Delayed entry & Unstable work & Mainly no work & Late entry \\
\hline Early entry & 1.00 & -0.67 & -0.33 & -0.34 & -0.75 \\
Delayed entry & -0.67 & 1.00 & 0.11 & -0.22 & 0.57 \\
Unstable work & -0.33 & 0.11 & 1.00 & -0.32 & 0.07 \\
Mainly no work & -0.34 & -0.22 & -0.32 & 1.00 & 0.01 \\
Late entry & -0.75 & 0.57 & 0.07 & 0.01 & 1.00 \\
\hline
\end{tabular}

Table A.7: Correlation coefficients between cluster representativeness

\begin{tabular}{rrrrrr}
\hline & Early entry & Delayed entry & Unstable work & Mainly no work & Late entry \\
\hline Early entry & 1.00 & 0.50 & 0.82 & -0.95 & -0.06 \\
Delayed entry & 0.50 & 1.00 & 0.57 & -0.32 & 0.73 \\
Unstable work & 0.82 & 0.57 & 1.00 & -0.71 & 0.16 \\
Mainly no work & -0.95 & -0.32 & -0.71 & 1.00 & 0.26 \\
Late entry & -0.06 & 0.73 & 0.16 & 0.26 & 1.00 \\
\hline
\end{tabular}

